\title{
3D Heat Transfer Analysis of a Miniature Copper-Water Vapor Chamber with Wicked Pillars Array
}

\author{
Yong Jiang, ${ }^{1}$ Gerardo Carbajal,, ${ }^{2}$. B. Sobhan, ${ }^{3}$ and Ji Li ${ }^{4}$ \\ ${ }^{1}$ College of Physics, University of Chinese Academy of Sciences, 19A Yuquanlu Road, Shijingshan District, Beijing 100049, China \\ ${ }^{2}$ Department of Mechanical Engineering, University of Turabo, Gurabo, PR 00778, USA \\ ${ }^{3}$ Department of Mechanical Engineering, National Institute of Technology, Calicut, Kerala 673 601, India \\ ${ }^{4}$ Laboratory of Advanced Thermal Management Technologies, College of Physics, University of Chinese Academy of Sciences, \\ 19A Yuquanlu Road, Shijingshan District, Beijing 100049, China
}

Correspondence should be addressed to Ji Li; dr.jimli@gmail.com

Received 24 January 2013; Accepted 11 February 2013

Academic Editors: S. W. Chang, P. Dineva, K. Ismail, G. Juncu, and P. Reis

Copyright (c) 2013 Yong Jiang et al. This is an open access article distributed under the Creative Commons Attribution License, which permits unrestricted use, distribution, and reproduction in any medium, provided the original work is properly cited.

A three-dimensional analysis of the heat and mass transfer phenomena inside a vapor chamber is essential for correctly understanding its thermal performance limitations and structural optimization. This paper presents a complete three-dimensional numerical analysis and comparative study of two different miniature vapor chambers designs with identical external geometry and dimensions but different internal structures: one having a wicked pillar array and the other one without the wicked pillars array. The distribution of the wicked pillar array in the vapor core was aligned. Detailed comparative experimental results are also reported, which were performed to verify the calculations from the numerical simulations. It was found that the numerical and experimental results agree quite well, especially at high heat flux values. It is also observed that the vapor chamber with wicked pillars had a better thermal performance than the conventional design, with a 5\% decrease in terms of total thermal resistance due to the added extra channels that allow a better flow of the working fluid to the evaporator surface. An insight into how improving the thermal performance of a vapor chamber is provided through the detailed three-dimensional numerical simulations.

\section{Introduction}

The recent developments in information technology demand large scale integration of electronic circuits, as well as better performance of microelectronic devices. However, as the heat generation rates in electronic chips are continuously becoming larger, to cool such systems effectively also has become a critical problem. Putra et al. [1] suggested that the heat flux of new microprocessor for commercial applications may exceed $100 \mathrm{~W} / \mathrm{cm}^{2}$. For hybrid starter-alternator in the automotive industry the electronic components can generate heat fluxes in the range $40-400 \mathrm{~W} / \mathrm{cm}^{2}$ [2]. Traditional cooling methods suggest increasing the total surface area of the heat sink or improving the forced convection with the use of fans, but these methods have a performance limit within a specified space constraint. Heat pipe technology is another commonly implemented method which has a number of industrial and electronic applications. Mochizuki et al. [3, 4] suggested the use of the vapor chamber is one of the most effective methods to cool chips. A vapor chamber, a special type of heat pipe, is a vacuum container with a wick structure lining the internal wall, which contains a working fluid, such as water. On applying heat, the water evaporates at the heated surface, and the vapor condenses back to the liquid state dissipating the heat to external environment at the cooling surface. The condensed liquid flows back to the evaporator section through the wick structure, by capillary effect.

Analysis of the vapor chamber has been reported in the literature [5-9]. Koito et al. [5] investigated the thermal resistance in a vapor chamber, considering different experimental parameters such as the heat flux, the ambient temperature, and the orientation angle. Boukhanouf et al. [6] presented results on the thermal spreading performance of a vapor chamber, by performing experimental studies using an infrared (IR) thermal imaging camera. Uniform temperature distribution obtained on the evaporator surface of the vapor 
chamber indicated excellent heat spreading effect, with different heat flux inputs. Xuan et al. [7] reported and discussed a new simplified two-dimensional model developed for a vapor chamber. Koito et al. [8] designed a vapor chamber with a large wicked pillar placed at its center and carried out numerical investigations of heat and fluid flow with an axial-symmetric model. The velocity, pressure, and temperature distributions inside the vapor chamber were explored by solving the equations of continuity, momentum, and energy conservation. Hsieh et al. [9] have presented a threedimensional numerical model to predict the temperature distributions and heat transfer in a vapor chamber. Carbajal et al. [10] performed a detailed analysis of the thermal response of a vapor chamber subjected to a nonuniform heat input. It was found that the vapor chamber spread the heat input uniformly at the condenser side. A similar study was also performed using a quasi-3D numerical modeling [11]. Queheillalt et al. [12] proposed a vapor chamber with crossshaped honeycomb truss. They found that the presence of the truss in the core can improve the thermal spreading capabilities of the system. Several numerical or analytical studies on flat heat pipes have also been reported in the literature [13-17].

Based on patents review, a miniature cylindrical pillar array was proposed to be applied in the core of a vapor chamber, commercially. However, there was no any detailed heat transfer analysis for such a vapor chamber with a wicked pillars array. It is expected that the miniature vapor chamber design with multiple wicked pillars sandwiched between the main wick structures inside the vacuum container can improve the thermal performance of a vapor chamber in principle. These wicked pillars play two important roles: as structural support and also as water reservoir with more number of channels that allow a better flow of the working fluid to the evaporator surface. This study compares the thermal resistances in a vapor chamber with and without wicked pillars, thus providing clear understanding of the thermal characteristics of a vapor chamber and the effects of the wicked pillars. Additionally, detailed numerical results for the three-dimensional velocity, pressure, and temperature distributions inside the vapor chambers are presented. The numerical simulations were also compared against the experimental data.

\section{Description of the Vapor Chamber}

A vapor chamber utilizes a working fluid which circulates through a porous wick material, and so the wick structure is of prime importance in the design, especially as it has to be accommodated in a limited space. In the present work, as mentioned before, two types of wick structures have been considered for analysis and comparison. One of these two designs has sintered copper pillars, and the other one is a conventional vapor chamber without the copper pillars. Figure 1 schematically shows the geometry and structure of the proposed miniature vapor chamber with wicked pillars. There are two advantages for this structure.
(1) The wicked pillars can enhance the structural support of the vapor chamber. Under the vacuum conditions inside the vapor chamber, this design ensures that the shape of the device is preserved, as also the walls do not undergo any thermal stress deformation under heating.

(2) The wicked pillar array increases the amount of working fluid. This design adds more flow paths available for the working fluid to pump it back to the evaporation area, so that the flow resistance (or pressure drop) of the working fluid is reduced during this process. This effect is especially useful in limited space, if the vapor chamber has a compact inner structure with a very thin layer of main wick. Specifications of the structure of the two vapor chambers design analyzed in the present work are shown in Table 1.

\section{Mathematical Model and Numerical Solution}

The analysis was performed by solving the system of governing equations, as proposed in the literature by Boukhanouf et al. [6] for the vapor chamber. The domain of analysis was appropriately selected to accommodate the wick structure including the pillar array in the proposed design.

3.1. Governing Equations. The mass conservation, momentum, and energy equations were applied as required for the analysis of the vapor chamber components. Due to the complexity of the problem, the following assumptions were made.

(i) Steady-state process.

(ii) Laminar flow in the vapor chamber.

(iii) The porous medium structure is homogeneous and isotropic.

(iv) The wick structure is fully saturated with liquid at all the time.

(v) The condensation and evaporation processes of the working medium occur at the interface between the vapor and liquid-wick regions.

(vi) The thermal resistances of condensation and evaporation processes are negligible.

(vii) The radiative and gravitational effects are negligible.

(viii) Incompressible flow in the vapor and wick region.

Based on the sketch presented in Figure 2, there are three distinct regions for analysis: namely, the vapor region, the liquid-wick region, and the solid wall region. The mass conservation, the momentum, and energy equations pertaining to these three regions are written separately as follows. 
TABLE 1: Specifications of the structure of the two vapor chambers.

\begin{tabular}{lll}
\hline & With wicked pillars & Without wicked pillars \\
\hline Material of chamber & Copper & Copper \\
Material of wick & Sintered copper powder & Sintered copper powder \\
Working medium & Distilled water & Distilled water \\
Size of chamber & $80 \mathrm{~mm} \times 80 \mathrm{~mm} \times 4 \mathrm{~mm}$ & $80 \mathrm{~mm} \times 80 \mathrm{~mm} \times 4 \mathrm{~mm}$ \\
Thickness of chamber & $0.8 \mathrm{~mm}$ & $0.8 \mathrm{~mm}$ \\
Thickness of wick & $0.5 \mathrm{~mm}$ & $0.5 \mathrm{~mm}$ \\
Area of heat source & $25 \mathrm{~mm} \times 25 \mathrm{~mm}$ & $25 \mathrm{~mm} \times 25 \mathrm{~mm}$ \\
Area of cooling surface & $70 \mathrm{~mm} \times 70 \mathrm{~mm}$ & $70 \mathrm{~mm} \times 70 \mathrm{~mm}$ \\
Diameter of wicked pillars & $5 \mathrm{~mm}$ & - \\
Height of wicked pillars & $1.4 \mathrm{~mm}$ & - \\
Number of wicked pillars & 36 & - \\
\hline
\end{tabular}

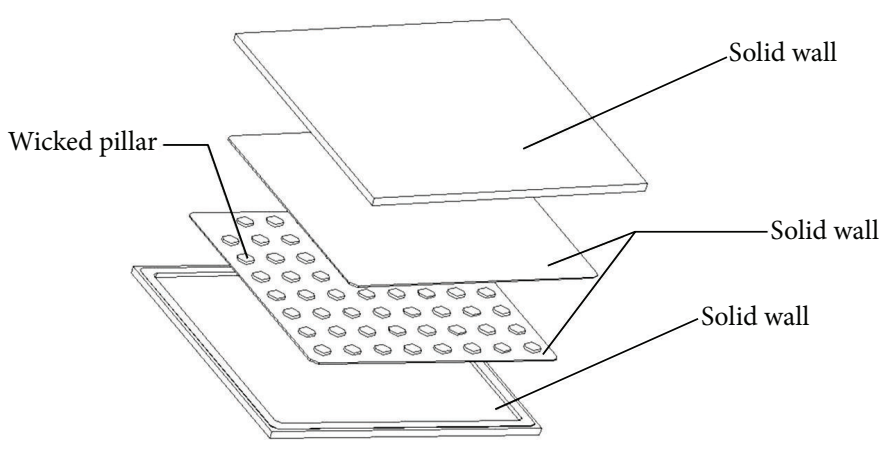

(a)

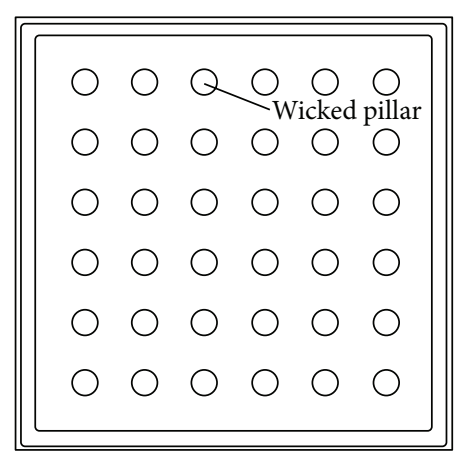

(b)

FIgURE 1: The structure of the proposed miniature vapor chamber with wicked pillars.

For the vapor region,

$$
\begin{gathered}
\nabla \cdot\left(\rho_{v} \vec{U}_{v}\right)=0, \\
\nabla \cdot\left(\rho_{v} \vec{U}_{v} \vec{U}_{v}\right)=-\nabla p_{v}+\nabla \cdot\left(\mu_{v} \nabla \vec{U}_{v}\right), \\
\nabla \cdot\left[\left(\rho_{v} c_{p, v}\right) \vec{U}_{v} T_{v}\right]=\nabla \cdot\left(k_{v} \nabla T_{v}\right) .
\end{gathered}
$$

For the liquid-wick region,

$$
\begin{gathered}
\nabla \cdot\left(\rho_{l} \vec{U}_{l}\right)=0, \\
\nabla \cdot\left(\rho_{l} \vec{U}_{l} \vec{U}_{l}\right)=-\varepsilon \nabla p_{v}+\nabla \cdot\left(\mu_{l} \nabla \vec{U}_{l}\right) \\
-\frac{\varepsilon \mu_{l}}{K} \vec{U}_{l}-\frac{2 \sigma \cos \theta}{r_{\text {cap }}^{2}} \nabla r_{\text {cap }} \cdot \nabla r, \\
\nabla \cdot\left[\left(\rho_{l} c_{p, l}\right) \vec{U}_{l} T_{l}\right]=\nabla \cdot\left(\frac{k_{\text {eff }}}{\varepsilon} \nabla T_{l}\right) .
\end{gathered}
$$

In (2b), Brickman and Forchheimer terms were neglected. The capillarity effect is included in the last term on the right hand side of this equation.

For the solid wall region,

$$
\nabla \cdot\left(k_{s} \nabla T_{s}\right)=0
$$

where $k_{s}$ is the thermal conductivity of the solid wall and $T_{s}$ is the solid wall temperature.

The permeability of the porous structure, $K$, used in (2b) can be calculated directly for the sintered wick manufactured from spherical metal powder using [16]

$$
K=\frac{d^{2} \cdot \varepsilon^{3}}{150(1-\varepsilon)^{2}} .
$$

The effective thermal conductivity of the liquid-wick sintered from spherical metal powder can be determined by using Maxwell's model [15]:

$$
k_{\text {eff }}=\frac{k_{l}\left[\left(2 k_{l}+k_{w}\right)-2(1-\varepsilon)\left(k_{l}-k_{w}\right)\right]}{\left[\left(2 k_{l}+k_{w}\right)+(1-\varepsilon)\left(k_{l}-k_{w}\right)\right]},
$$

where $d$ is the diameter of the copper powder, $k_{l}$ is the liquid thermal conductivity, and $k_{w}$ is the thermal conductivity of the wick material.

In this analysis it was considered that the thermal conductivity, density, specific heat, and the dynamic viscosity of the working fluid are all constant.

3.2. The Boundary Conditions. Five types of boundaries can be identified in the computational domain of the vapor chamber, as depicted in Figure 2. These are the heat source, the water cooled surface, the adiabatic peripheral surfaces, 


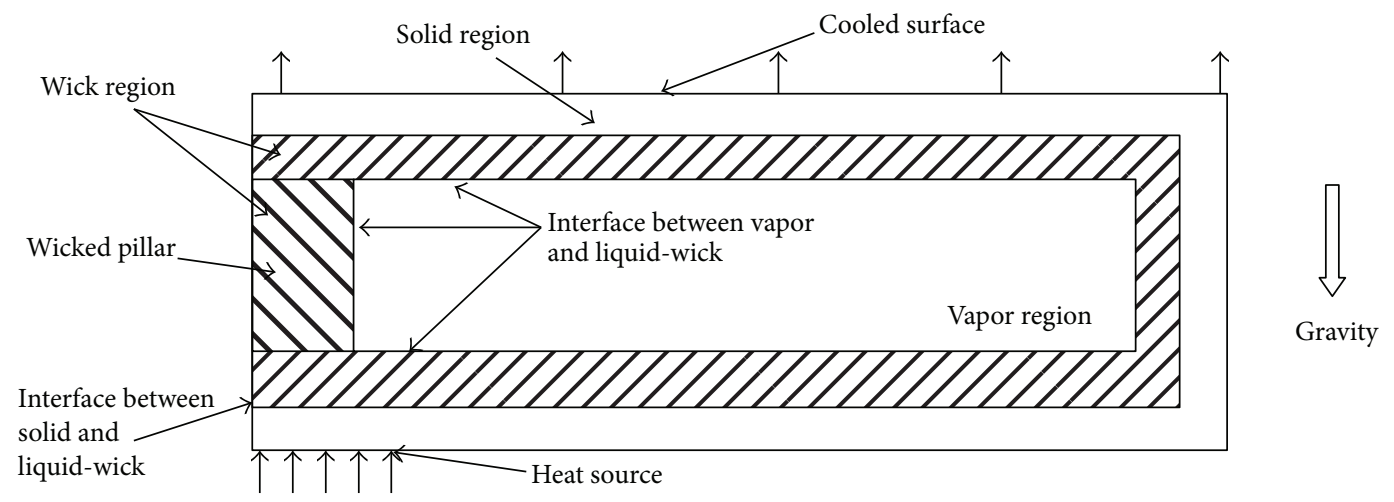

FIGURE 2: Schematic of the numerical domain and the boundary conditions.

the interfaces between the liquid-wick region and the solid wall, and the interfaces between the vapor region and the liquid-wick region. The boundary conditions are expressed mathematically as follows $[8,18]$.

For the heat source,

$$
-k_{s} \frac{\partial T_{s}}{\partial z}=q
$$

For the water cooled surface,

$$
-k_{s} \frac{\partial T_{s}}{\partial z}=h\left(T_{s}-T_{\text {water }}\right)
$$

where $T_{\text {water }}$ is the cooling water temperature.

For the adiabatic outer surfaces,

$$
-k_{s} \nabla T_{s}=0
$$

For the interfaces between the liquid-wick and the solid wall, assuming perfect contact between the two surfaces (no contact thermal resistance),

$$
\begin{gathered}
\vec{U}_{l}=0 \\
k_{s} \frac{\partial T_{s}}{\partial x}=k_{\text {eff }} \frac{\partial T_{l}}{\partial x} \\
k_{s} \frac{\partial T_{s}}{\partial y}=k_{\text {eff }} \frac{\partial T_{l}}{\partial y} \\
k_{s} \frac{\partial T_{s}}{\partial z}=k_{\text {eff }} \frac{\partial T_{l}}{\partial z} .
\end{gathered}
$$

For the interfaces between the vapor and the liquid-wick,

$$
\begin{gathered}
T=T_{\text {sat }}, \\
\mu_{l} \frac{\partial u_{l}}{\partial x}=\mu_{v} \frac{\partial u_{v}}{\partial x}, \\
\mu_{l} \frac{\partial v_{l}}{\partial y}=\mu_{v} \frac{\partial v_{v}}{\partial y} \\
\mu_{l} \frac{\partial w_{l}}{\partial z}=\mu_{v} \frac{\partial w_{v}}{\partial z} .
\end{gathered}
$$

The thickness of the liquid-vapor interface was assumed to be negligible. This interface did not absorb/store energy. Also, it was considered a perfect evaporation and condensation process, respectively. Based on these considerations the following conditions were applied at the interface:

$$
\begin{aligned}
& \rho_{v} u_{v}=\rho_{l} u_{l}=\frac{1}{h_{\mathrm{fg}}}\left(k_{v} \frac{\partial T_{v}}{\partial x}-k_{\mathrm{eff}} \frac{\partial T_{l}}{\partial x}\right), \\
& \rho_{v} v_{v}=\rho_{l} v_{l}=\frac{1}{h_{\mathrm{fg}}}\left(k_{v} \frac{\partial T_{v}}{\partial y}-k_{\mathrm{eff}} \frac{\partial T_{l}}{\partial y}\right), \\
& \rho_{v} w_{v}=\rho_{l} w_{l}=\frac{1}{h_{\mathrm{fg}}}\left(k_{v} \frac{\partial T_{v}}{\partial z}-k_{\mathrm{eff}} \frac{\partial T_{l}}{\partial z}\right) .
\end{aligned}
$$

In the above equations, $u_{v}, v_{v}$, and $w_{v}$ are the vapor velocity components and $u_{l}, v_{l}$, and $w_{l}$ are the liquid velocity components, in the $x, y$, and $z$ directions, respectively. The latent heat of water is represented by $h_{\mathrm{fg}}$ and assumed as a constant parameter.

3.3. The Numerical Solution. The CFD commercial solver, Fluent, was used to obtain the numerical solutions for the fluid flow and heat transfer. The three-dimensional temperature and velocity distributions in the computational field were obtained from the calculations. The convergence criterion was set as

$$
\begin{aligned}
& \sum \sum \sum\left|T_{\text {new }}(i, j, k)-T_{\text {old }}(i, j, k)\right| \leq 10^{-6}, \\
& \sum \sum \sum\left|P_{\text {new }}(i, j, k)-P_{\text {old }}(i, j, k)\right| \leq 10^{-7} .
\end{aligned}
$$

The total grid number chosen was 234,000 for the vapor chamber without pillars and 367,000 for the vapor chamber with pillars.

\section{Results and Discussion}

A numerical simulation was performed, with different heat inputs, to determine the effect of the wicked pillars on the velocity field and the temperature distribution in the vapor chamber. Further, in this work, in order to benchmark the results, an experimental study was carried out with a 
fabricated vapor chamber, with specifications listed in Table 1. The sketch of the experimental vapor chamber utilized for this analysis is illustrated in Figure 3. The experimental vapor chamber was cooled by circulating water over its top surface.

Figures 4 and 5 show the numerical results for the temperature distribution in the vapor chamber without wicked pillars and with wicked pillars, respectively, for a heat input of $q=23 \mathrm{~W} / \mathrm{cm}^{2}$. Referring to Figures 4(a) and 5(a) for the evaporator surface, the maximum temperature occurs at the central point of the bottom surface. At steady state, as considered in the present analysis, the vapor chamber with wicked pillar array is found to have a lower temperature at the center of the evaporation surface than that in the conventional design, indicating a reduction of the maximum temperature in the proposed design. It is also noted that the vapor chamber with wicked pillars has a more uniform temperature distribution at the evaporation surface than the design without pillar array. Both of these are desirable effects, especially in applications related to thermal management of electronics cooling.

The above observations can be attributed to the fact that the wicked pillars in the vapor chamber can enhance the rate of fluid circulation to the evaporator and condenser sides, respectively, thus increasing heat spreading effects. As Figure 5(a) shows, the zone around the pillars has the lowest temperature. With an input heat flux $q=23 \mathrm{~W} / \mathrm{cm}^{2}$, the center of the evaporation surface is found to have a temperature of $46^{\circ} \mathrm{C}$. The temperature gradient along the condensation surface is very small, and the temperature difference between center and side edge was less than $1^{\circ} \mathrm{C}$. Thus, the vapor chamber with wicked pillars shows a better thermal performance. It is also observed that the temperature of the condensation surface of the vapor chamber filled with the pillar array was much more uniform than that without the pillars, implying that the proposed design is capable of spreading more uniformly the high heat flux input at the condensation surface.

Figure 6 shows the numerical results for the velocity distribution in the vapor chamber without the wicked pillar array for a heat flux input of $q=23 \mathrm{~W} / \mathrm{cm}^{2}$. Figures $6(\mathrm{a})$ and $6(\mathrm{~b})$, respectively, show the velocity distributions in the wick (cross-sectional view) and vapor region (top view). The maximum velocity in the wick, as well as in the vapor flow, occurs at the region surrounding the heated zone on the bottom surface. At this condition the maximum velocity of vapor in the vapor core was approximately $3.19 \mathrm{~m} / \mathrm{s}$. The maximum velocity in the wick was found to be $0.00475 \mathrm{~m} / \mathrm{s}$ approximately. It is clearly seen that the vapor velocity decreases beyond the heated area. This is because the vapor starts to condense at the colder zone along the condenser area; thus the magnitude of the mass flow rate and the vapor velocity reduces progressively outside the heated area.

The numerical results for the velocity distribution in the vapor chamber filled with a wicked pillar array, for the same heat input, are shown in Figure 7. Figures 7(a) and 7(b) show the cross- and top-sectional view of the velocity vector distribution in the vapor region. For the vapor chamber with wicked pillars, for the same heat input, the maximum vapor velocity obtained was $4 \mathrm{~m} / \mathrm{s}$, which was approximately 25 percent larger than that in the case without the wicked pillar array. The location of the maximum vapor velocity is found to be near the center of the vapor chamber. From Figure 7(a), it can be inferred that the vapor flow bypasses the pillars and condenses, and, correspondingly, the mass flow rate reduces along the flow to the condenser side. Figures $7(\mathrm{c})$ and $7(\mathrm{~d})$ show the velocity distributions in the wicked pillars at the middle cross-section of the vapor chamber. It can be understood that most of the working fluid returns through the wicked pillars, and so it can be concluded that the wicked pillars can provide additional path for the working fluid, thus increasing the rate and reducing the flow resistance for circulation of the working fluid.

Figures $8(\mathrm{a})$ and $8(\mathrm{~b})$ show the cross-sectional velocity distributions of the working fluid at the evaporating and condensing wick surfaces, respectively. It can be observed that evaporation and condensation occur mainly in the central portion of the vapor chamber. The velocity vector plot in Figure 8(b) shows that the working fluid flows from the top of wick to its bottom, which is in accordance with the actual path of the liquid in the physical domain.

\section{Comparative Results}

The numerical results from the numerical model for the vapor chamber with and without wicked pillar array were compared with computed results for a solid copper plate with the same dimensions, as well as with the actual experimental results from a fabricated vapor chamber. Observations from these comparative studies are explained below.

In order to evaluate the thermal performance of the vapor chamber of interest, a comparative study was first performed with computed results for a solid copper plate which has the same size as the vapor chamber. The thermal resistance, an important performance indicator for the vapor chamber, has been defined by Li and Peterson [17] as $R_{\mathrm{vc}}=\left(T_{\mathrm{vc}}-T_{\text {cool }}\right) / \mathrm{Q}$, where $T_{\mathrm{vc}}$ is the center temperature of vapor chamber, $T_{\text {cool }}$ is the temperature of the cooling water, and $Q$ is the heating power. A comparison of the thermal resistances of the vapor chamber and the copper plate has been performed to evaluate the performance of the vapor chamber.

Figures 9 and 10 show a comparative thermal performance of the two vapor chambers and the solid copper plate. The temperature response and the thermal resistances values for different heat loads were used as the performance indicators. Figure 9 shows the comparison of the numerical results for the temperature at the center of the evaporator surface for different heat loads. It is seen that this temperature is significantly lower in the case of the vapor chamber, compared to the copper plate.

When the heat load is increased, the difference in temperature between copper plate and vapor chambers becomes larger. At the heat load of $146 \mathrm{~W}$, the temperature at the center of the copper plate surface was nearly $368 \mathrm{~K}\left(95^{\circ} \mathrm{C}\right)$ which is pretty high for electronic cooling applications. However, the center temperature of the evaporator surface for the vapor chambers was only around $319 \mathrm{~K}\left(46^{\circ} \mathrm{C}\right)$. This temperature 


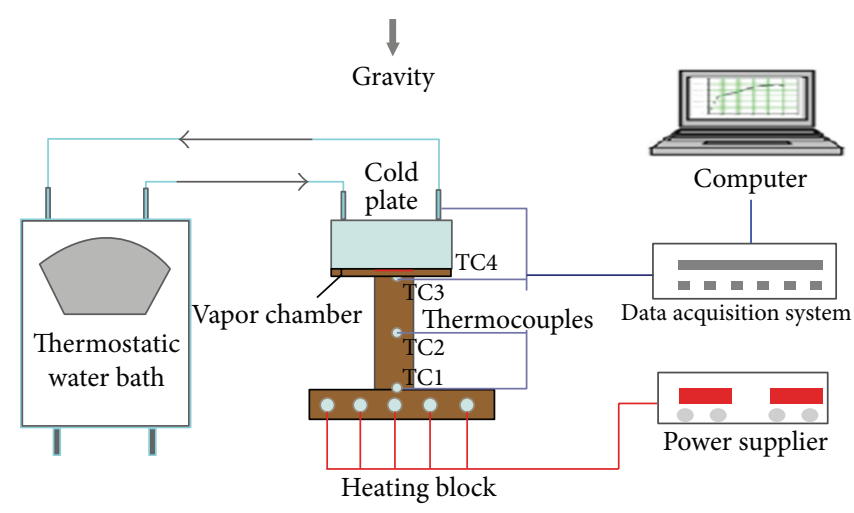

FIGURE 3: Schematic of the experimental system.

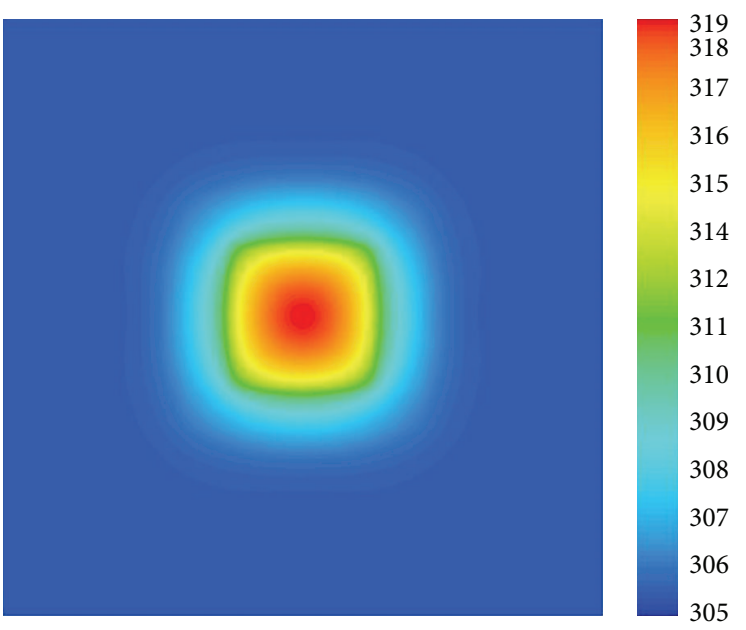

(a) Temperature distribution in the evaporator surface (without pillars)

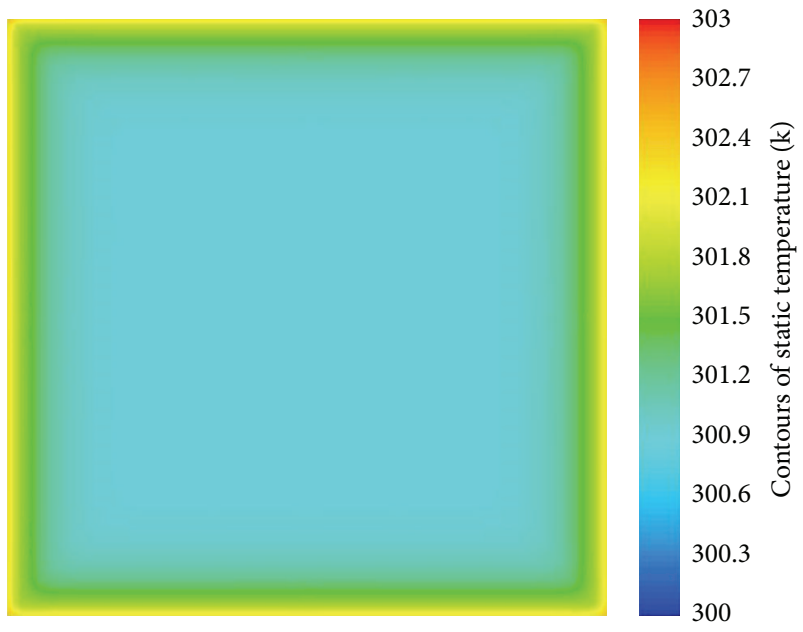

(b) Temperature distribution in the condenser surface (without pillars)

FIGURE 4: Temperature distribution in the vapor chamber without wicked pillars for $q=23 \mathrm{~W} / \mathrm{cm}^{2}$.

response clearly suggests that the vapor chamber has a much better thermal performance, with a temperature level suitable for thermal management of electronics, because it quickly transfers heat from the evaporator to the condenser by means of phase change as the underlying physical phenomenon. Further, the vapor chamber can function more effectively at higher heat flux input conditions, as it is observed from the temperature variation with respect to the heat load.

From Figure 10, the thermal resistance for the copper plate is found to be nearly $0.5^{\circ} \mathrm{C} / \mathrm{W}$, which is much higher than that for the vapor chambers, which were approximately $0.15^{\circ} \mathrm{C} / \mathrm{W}$.

The results presented in Figures 9 and 10 also clearly show that the vapor chamber with wicked pillars is more effective than the conventional one without wicked pillars. This effect can be attributed to the lower thermal resistance and center temperature attained by the proposed design with wicked pillars, at the same heat load.

In order to verify the accuracy of the numerical results and the ability of the model to correctly predict the performance of the vapor chamber, a comparison was performed between the computational results and experimental data $[18,19]$. Experiments were performed on a vapor chamber with a wicked pillar array, fabricated as per the details given in Table 1 . The test specimen was fastened onto a heating copper block of surface dimensions $25 \mathrm{~mm} \times 25 \mathrm{~mm}$. Heat flux inputs were supplied in the range between 5 and $23 \mathrm{~W} / \mathrm{cm}^{2}$, and the condensing surface of the vapor chamber was cooled down with water flow at an inlet temperature of $300 \mathrm{~K}$.

Figure 11 gives a comparison between the numerical results and the experimental results. The figure shows that the difference between the thermal resistance calculated from numerical results and obtained from the experimental results becomes smaller as the heat input increases. This behavior is because the experimental vapor chamber is not fully operational at lower heat fluxes. Though the numerical simulation assumes a fully saturated wick in the vapor chamber, at low heat flux inputs, practically the phase change phenomenon would not be adequate for a full-fledged operation. At higher heating levels, however, this limitation is overcome, and the theoretical and experimental values of the thermal resistance are found to match quite well. 


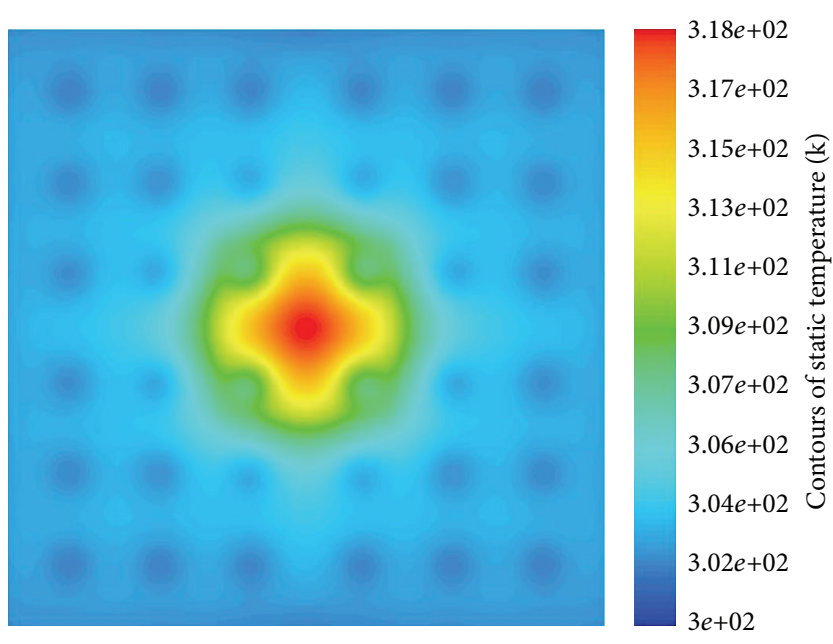

(a) Temperature distribution in the evaporator surface (with pillars)

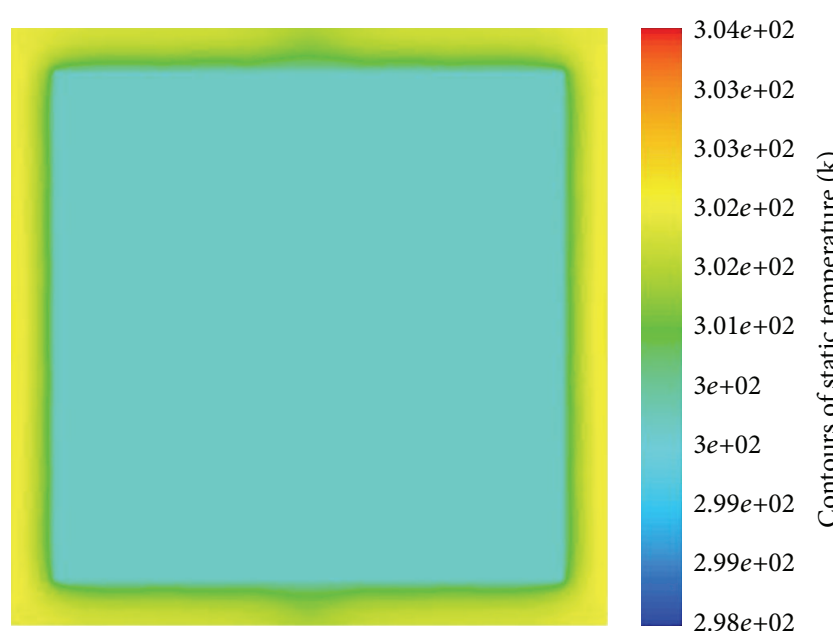

(b) Temperature distribution in the condenser surface (with pillars)

FIGURE 5: Temperature distribution in the vapor chamber with wicked pillars for $q=23 \mathrm{~W} / \mathrm{cm}^{2}$.

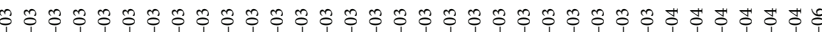

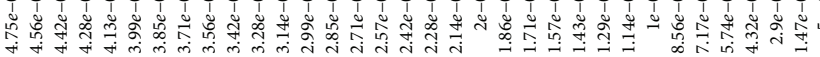

Velocity vectors colored by velocity magnitude $(\mathrm{m} / \mathrm{s})$

(a) Symmetric velocity distribution in the wick region (without pillars)
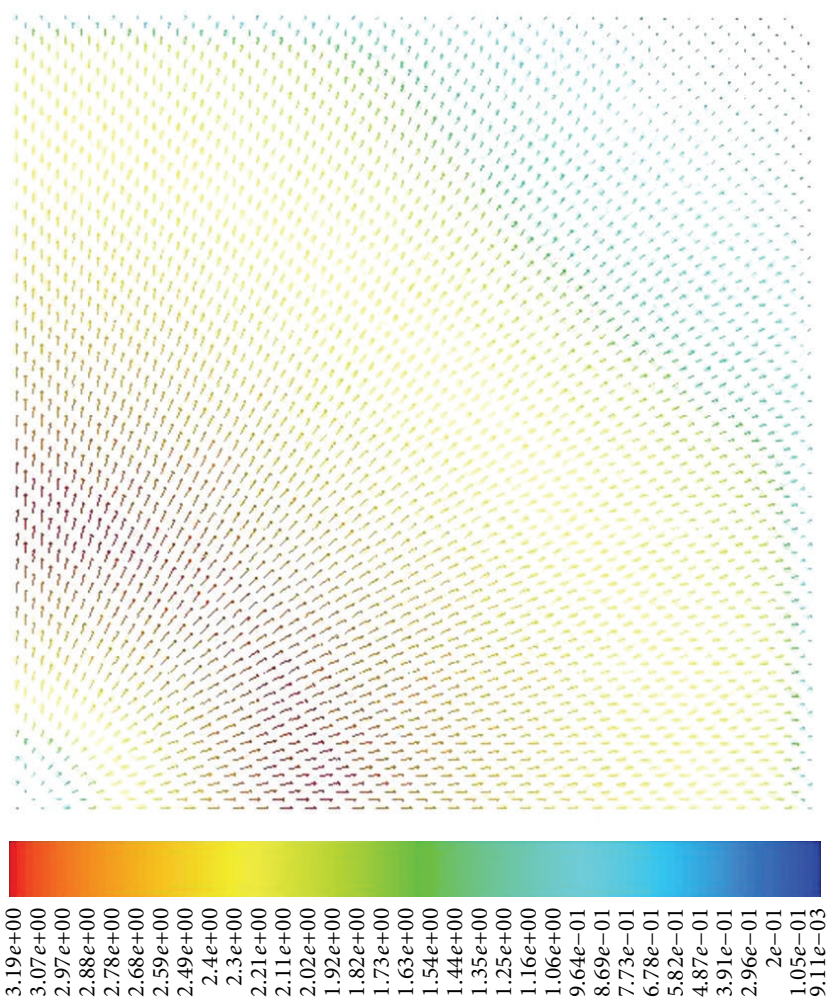

Velocity vectors colored by velocity magnitude $(\mathrm{m} / \mathrm{s})$

(b) Velocity distribution in the vapor region (without pillars)

FIGURE 6: Velocity distribution in the vapor chamber (without pillars) for $q=23 \mathrm{~W} / \mathrm{cm}^{2}$.

Due to the complicated two-phase flow in the vapor chamber filled with wicked pillars, as discussed in the previous section, further visualization studies are expected to verify the numerical results from low to high heat flux situations $[19,20]$.

\section{Conclusions}

A three-dimensional numerical model for fluid flow and heat transfer in vapor chambers with two different wick structures has been presented in this paper. Computational studies on 


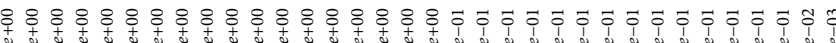

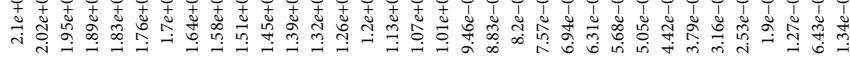
Velocity vectors colored by velocity magnitude $(\mathrm{m} / \mathrm{s})$

(a) Symmetric velocity distribution in the vapor region (with pillars)

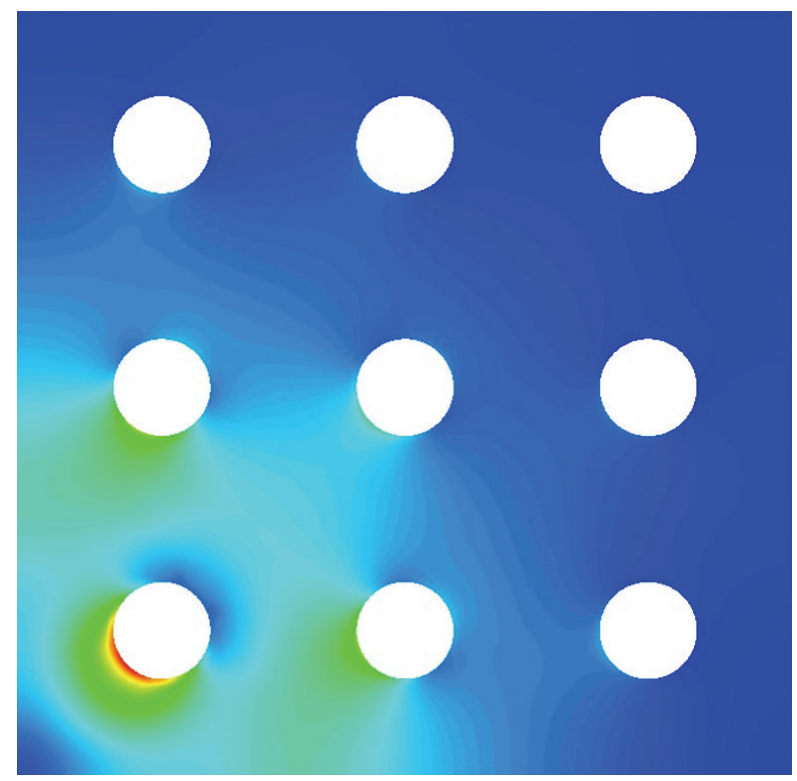

mp

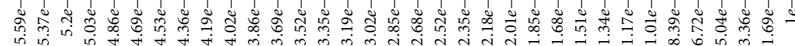

(c) Velocity distribution at the major evaporating wick surfaces

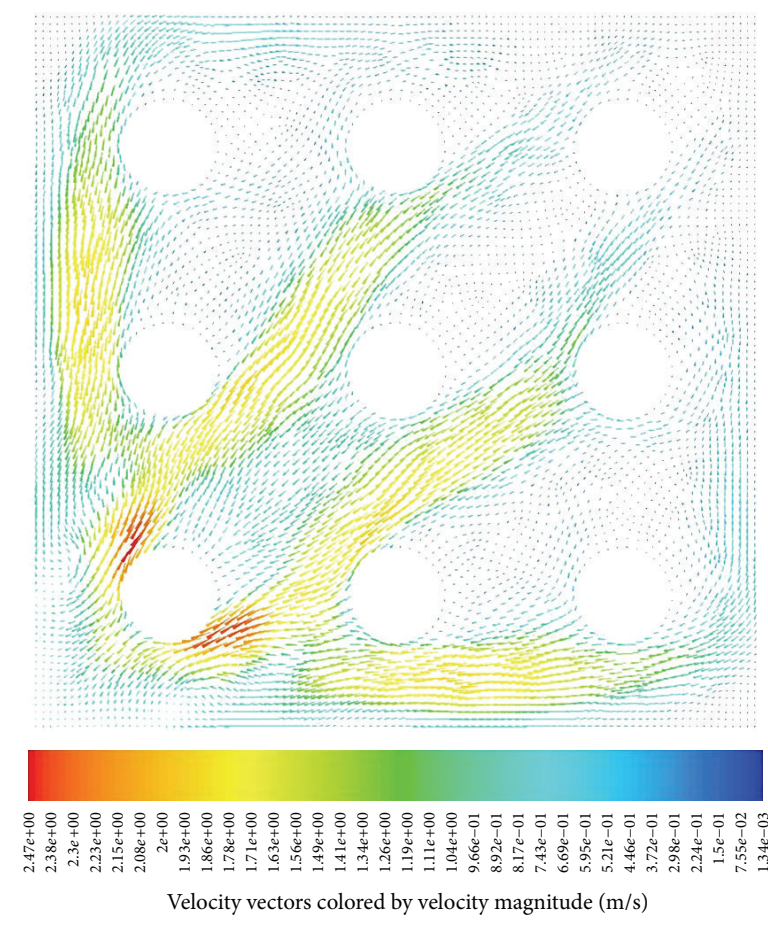

(b) Velocity distribution in the vapor region (with pillars)
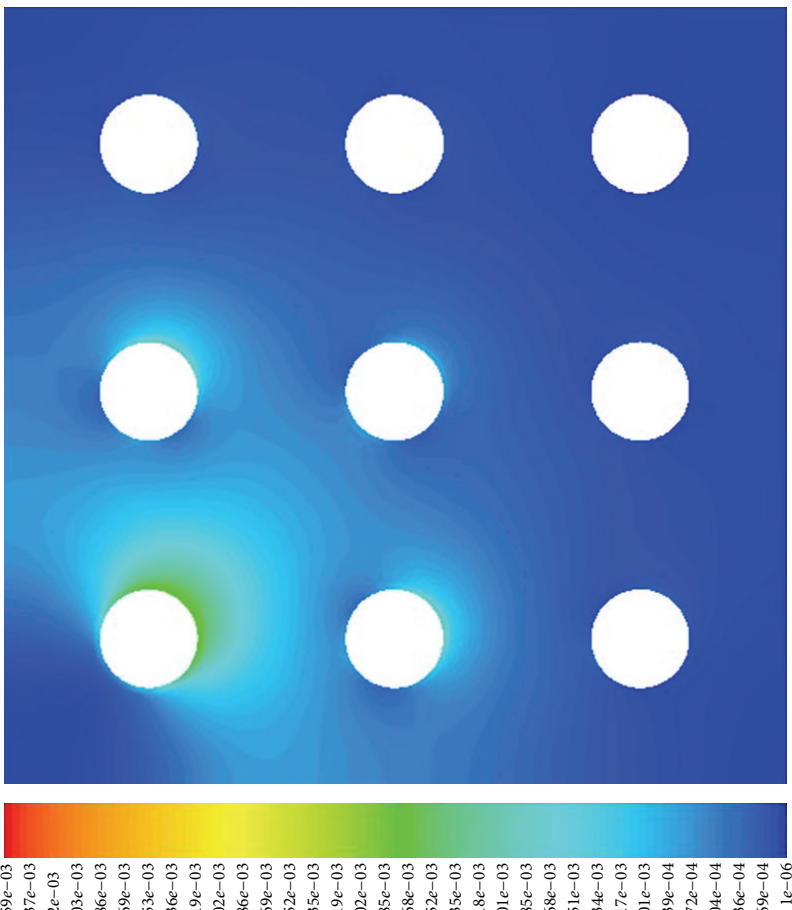

(d) Velocity distribution at the major condensing wick surfaces

FIGURE 7: Velocity distribution in the vapor chamber (with pillars) for $q=23 \mathrm{~W} / \mathrm{cm}^{2}$.

the performance of the vapor chambers have been performed using a CFD commercial software (fluent). The theoretical results were compared against experimental results for a fabricated vapor chamber with a novel design, which utilizes a wicked pillars array structure. Based on the numerical and experimental results, the following conclusions are drawn.
(1) Wicked pillars inside the vapor chamber provide more number of paths for working fluid circulation and hence make the vapor chamber a more effective heat spreader.

(2) The numerical results versus experimental results show larger difference at a low heat load, but the 


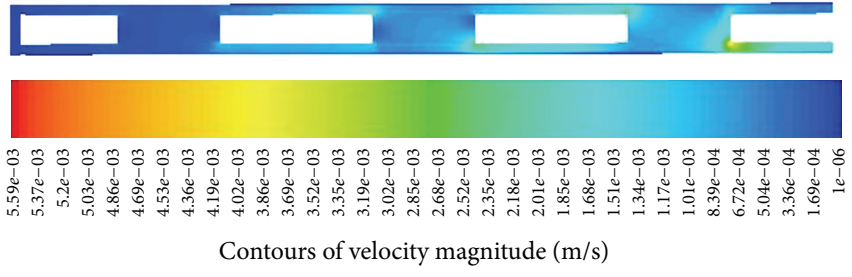

(a) Velocity distribution in the wicked pillars (an overall view)
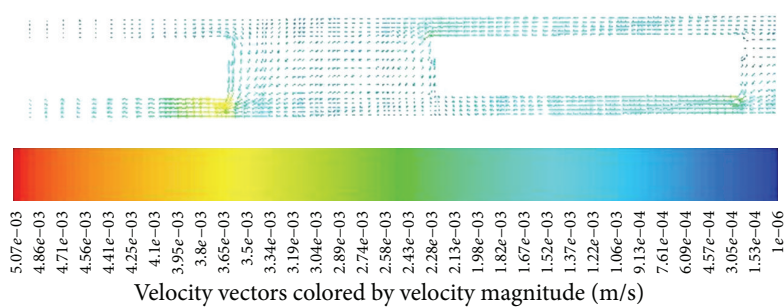

(b) Velocity distribution in the wicked pillars (an enlarged local view)

FIGURE 8: Liquid velocity distribution in the wicked pillars.

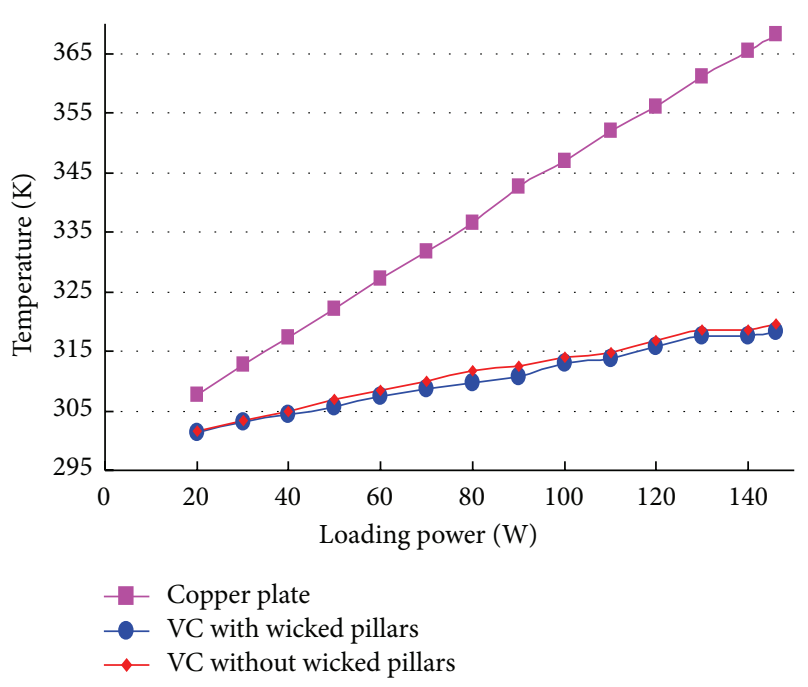

FIGURE 9: The center temperature at the bottom surface of the different cases under study, for different heat loads.

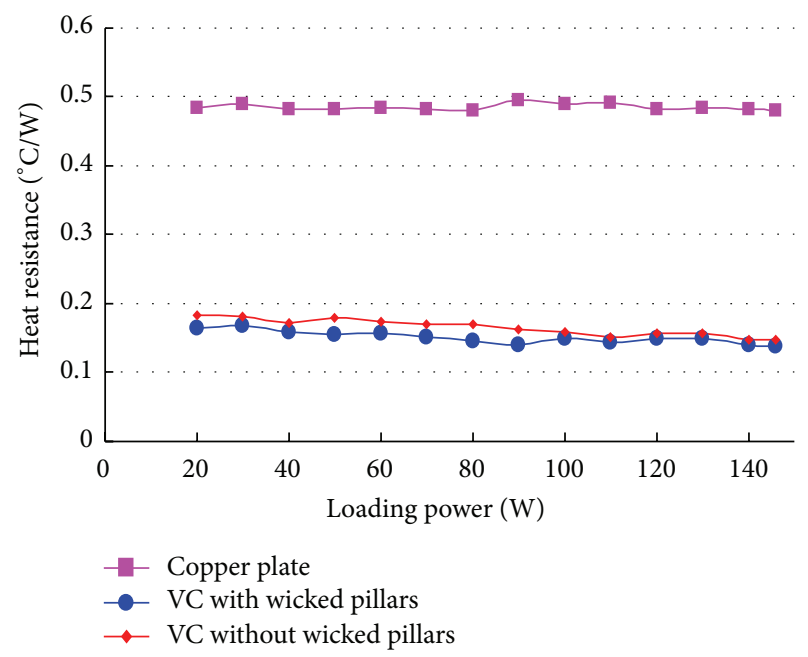

FIGURE 10: Thermal resistance for different heat loads.

results match well at high heat loads. This is because the experimental vapor chamber is not fully operational at low heat loads.

(3) The temperature distribution at the top side of the vapor chamber was found to be uniform, which

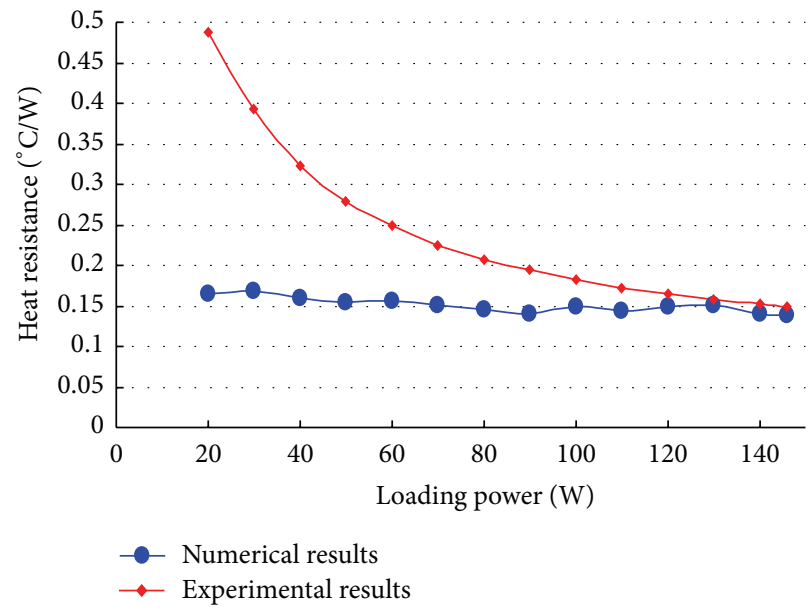

FIgURE 11: Comparison of thermal resistances from the numerical simulations and the experimental measurements, for different heat loads.

makes it suitable to be used as a high conductance heat spreader, especially in microelectronic applications.

(4) The temperature distribution on the top side of the vapor chamber filled with a wicked pillar array was five percent less than that of the vapor chamber without the wicked pillars.

\section{Nomenclature}

$C_{p}$ : Specific heat $\left(\mathrm{J} \mathrm{kg}^{-1} \mathrm{~K}^{-1}\right)$

$d$ : Diameter $(\mathrm{m})$

$h$ : Convection coefficient $\left(\mathrm{W} \mathrm{m}^{-2} \mathrm{~K}^{-1}\right)$

$h_{\mathrm{fg}}$ : Latent heat $\left(\mathrm{J} \mathrm{kg}^{-1}\right)$

$K$ : Permeability $\left(\mathrm{m}^{2}\right)$

$k$ : Thermal conductivity $\left(\mathrm{W} \mathrm{m}^{-1} \mathrm{~K}^{-1}\right)$

$P$ : Pressure $(\mathrm{Pa})$

Q: Heat transfer rate $(\mathrm{W})$

q: Heat flux $\left(\mathrm{W} \mathrm{m}^{-2}\right)$

$R:$ Thermal resistance $\left(\mathrm{K} \mathrm{W}^{-1}\right)$

$r$ : Vector, radial coordinate $(\mathrm{mm})$

$T: \quad$ Temperature (K)

$\vec{U}:$ Velocity vector $\left(\mathrm{m} \mathrm{s}^{-1}\right)$. 


\section{Greek Symbols}

\author{
$\varepsilon$ : Porosity \\ $\mu$ : Viscosity (Pa s) \\ $\rho$ : Density $\left(\mathrm{kg} \mathrm{m}^{-3}\right)$.
}

\section{Subscripts}

\author{
cap: Capillary \\ eff: Effective \\ exp: Experimental \\ $l$ : Liquid \\ $s$ : $\quad$ Solid \\ sat: Saturated \\ $v$ : Vapor region \\ vc: Vapor chamber \\ w: Wick.
}

\section{Acknowledgment}

This work was supported by the National Natural Science Foundation of China (Project no. 51176202).

\section{References}

[1] N. Putra, Yanuar, and F. N. Iskandar, "Application of nanofluids to a heat pipe liquid-block and the thermoelectric cooling of electronic equipment," Experimental Thermal and Fluid Science, vol. 35, no. 7, pp. 1274-1281, 2011.

[2] S. Harmand, R. Sonan, M. Fakès, and H. Hassan, "Transient cooling of electronic components by flat heat pipes," Applied Thermal Engineering, vol. 31, no. 11-12, pp. 1877-1885, 2011.

[3] M. Mochizuki, T. Nguyen, K. Mashiko et al., "Practical application of heat pipe and vapor chamber for cooling high performance personal computer," in Proceedings of the 13th International Heat Pipe Conference, pp. 448-454, 2004.

[4] M. Mochizuki, T. Nguyen, K. Mashiko et al., "Latest technology using micro heat pipes and vapor chamber for cooling personal computer," in Proceedings of the 1st International Symposium on Micro \& Nano Technology, 2004.

[5] Y. Koito, K. Motomatsu, H. Imura, M. Mochizuki, and Y. Saito, "Fundamental investigations on heat transfer characteristics of heat sinks with a vapor chamber," in Proceedings of the 7th International Heat Pipe Symposium, pp. 247-251, 2003.

[6] R. Boukhanouf, A. Haddad, M. T. North, and C. Buffone, "Experimental investigation of a flat plate heat pipe performance using IR thermal imaging camera," Applied Thermal Engineering, vol. 26, no. 17-18, pp. 2148-2156, 2006.

[7] Y. Xuan, Y. Hong, and Q. Li, "Investigation on transient behaviors of flat plate heat pipes," Experimental Thermal and Fluid Science, vol. 28, no. 2-3, pp. 249-255, 2004.

[8] Y. Koito, H. Imura, M. Mochizuki, Y. Saito, and S. Torii, "Numerical analysis and experimental verification on thermal fluid phenomena in a vapor chamber," Applied Thermal Engineering, vol. 26, no. 14-15, pp. 1669-1676, 2006.

[9] S. Hsieh, R. Lee, J. Shyu, and S. Chen, "Analytical solution of thermal resistance of vapor chamber heat sink with and without pillar," Energy Conversion and Management, vol. 48, no. 10, pp. 2708-2717, 2007.
[10] G. Carbajal, C. B. Sobhan, G. P. Peterson, D. T. Queheillalt, and H. N. G. Wadley, "Thermal response of a flat heat pipe sandwich structure to a localized heat flux," International Journal of Heat and Mass Transfer, vol. 49, no. 21-22, pp. 4070-4081, 2006.

[11] G. Carbajal, C. B. Sobhan, G. P. Bud Peterson, D. T. Queheillalt, and H. N. G. Wadley, "A quasi-3D analysis of the thermal performance of a flat heat pipe," International Journal of Heat and Mass Transfer, vol. 50, no. 21-22, pp. 4286-4296, 2007.

[12] D. T. Queheillalt, G. Carbajal, H. N. G. Wadley, and G. P. Peterson, "A multifunctional heat pipe sandwich panel structure," International Journal of Heat and Mass Transfer, vol. 51, no. 12, pp. 312-326, 2008.

[13] W. S. Chang and G. T. Colwell, " Mathematical modeling of the transient operating characteristics of a low-temperature heat pipe," Numerical Heat Transfer, vol. 8, no. 2, pp. 169-186, 1985.

[14] M. N. Chen and A. Faghri, "An analysis of the vapor flow and the heat conduction through the liquid-wick and pipe wall in a heat pipe with single or multiple heat sources," International Journal of Heat and Mass Transfer, vol. 33, no. 9, pp. 1945-1955, 1990.

[15] J. M. Tournier and M. S. El-Genk, "A heat pipe transient analysis model," International Journal of Heat and Mass Transfer, vol. 37, no. 5, pp. 753-762, 1994.

[16] Z. J. Zuo and A. Faghri, "Boundary element approach to transient heat pipe analysis," Numerical Heat Transfer A, vol. 32, no. 3, pp. 205-220, 1997.

[17] J. Li and G. P. Peterson, "3D heat transfer analysis in a loop heat pipe evaporator with a fully saturated wick," International Journal of Heat and Mass Transfer, vol. 54, no. 17-18, pp. 564574, 2011.

[18] J. Li, G. P. Peterson, and P. Cheng, "Three-dimensional analysis of heat transfer in a micro-heat sink with single phase flow," International Journal of Heat and Mass Transfer, vol. 47, no. 1920, pp. 4215-4231, 2004.

[19] J. Qu and H. Y. Wu, "Flow visualization of silicon-based micro pulsating heat pipes," Science China Technological Sciences, vol. 53, no. 4, pp. 984-990, 2010.

[20] J.-T. Feng, G.-P. Lin, and L.-Z. Bai, "Experimental investigation on operating instability of a dual compensation chamber loop heat pipe," Science in China E, vol. 52, no. 8, pp. 2316-2322, 2009. 

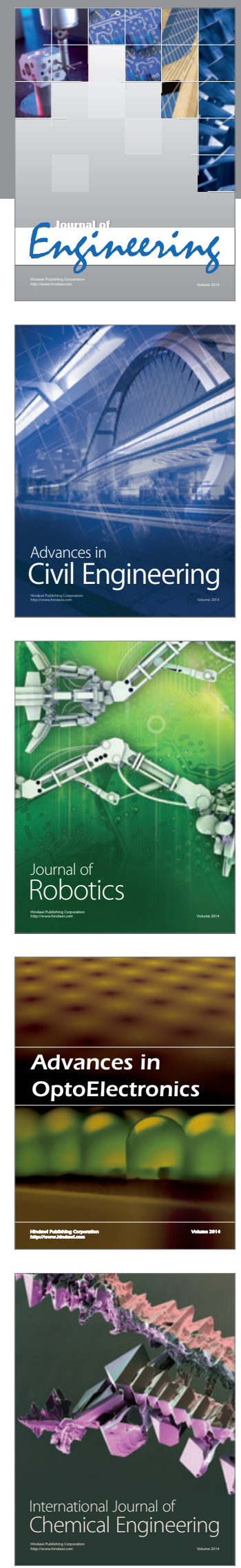

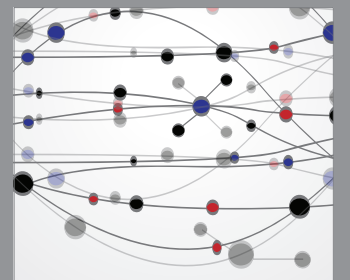

The Scientific World Journal
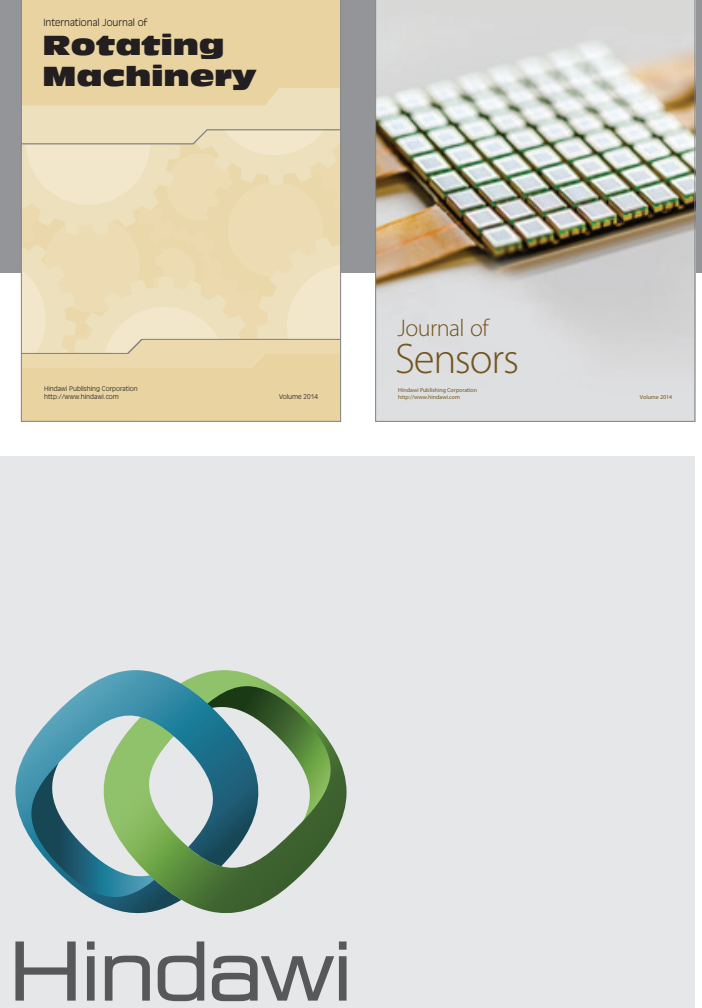

Submit your manuscripts at http://www.hindawi.com
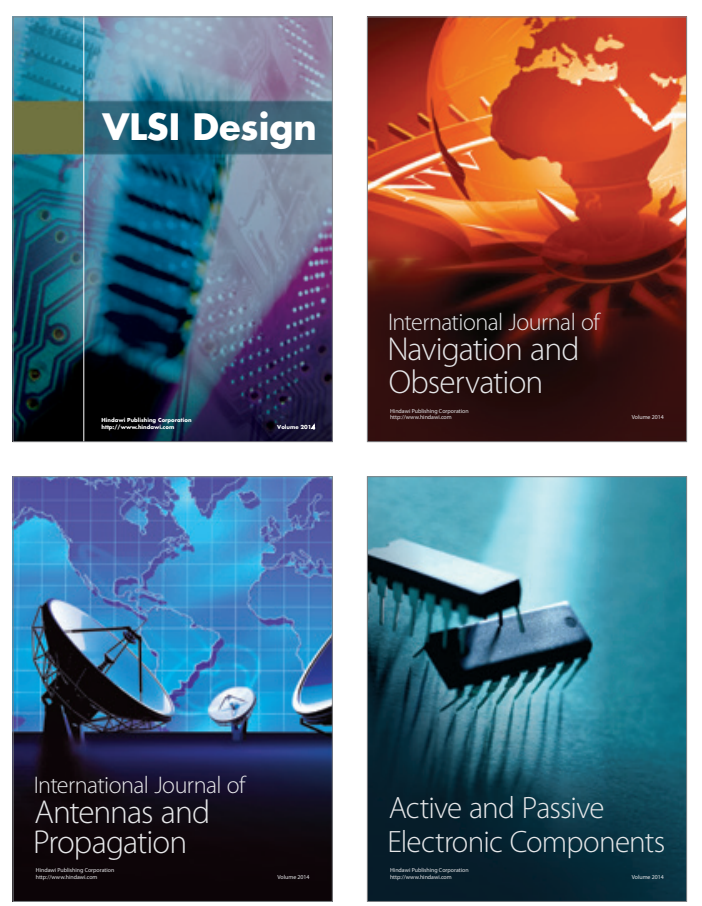
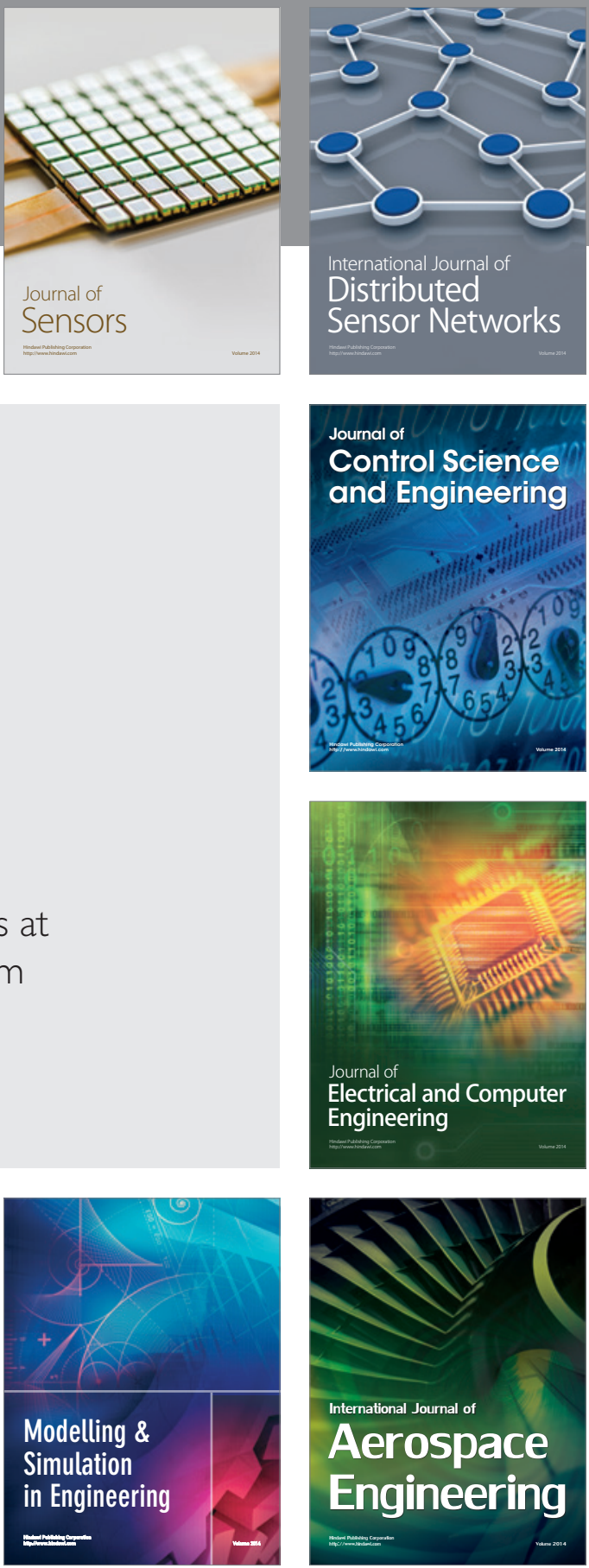

Journal of

Control Science

and Engineering
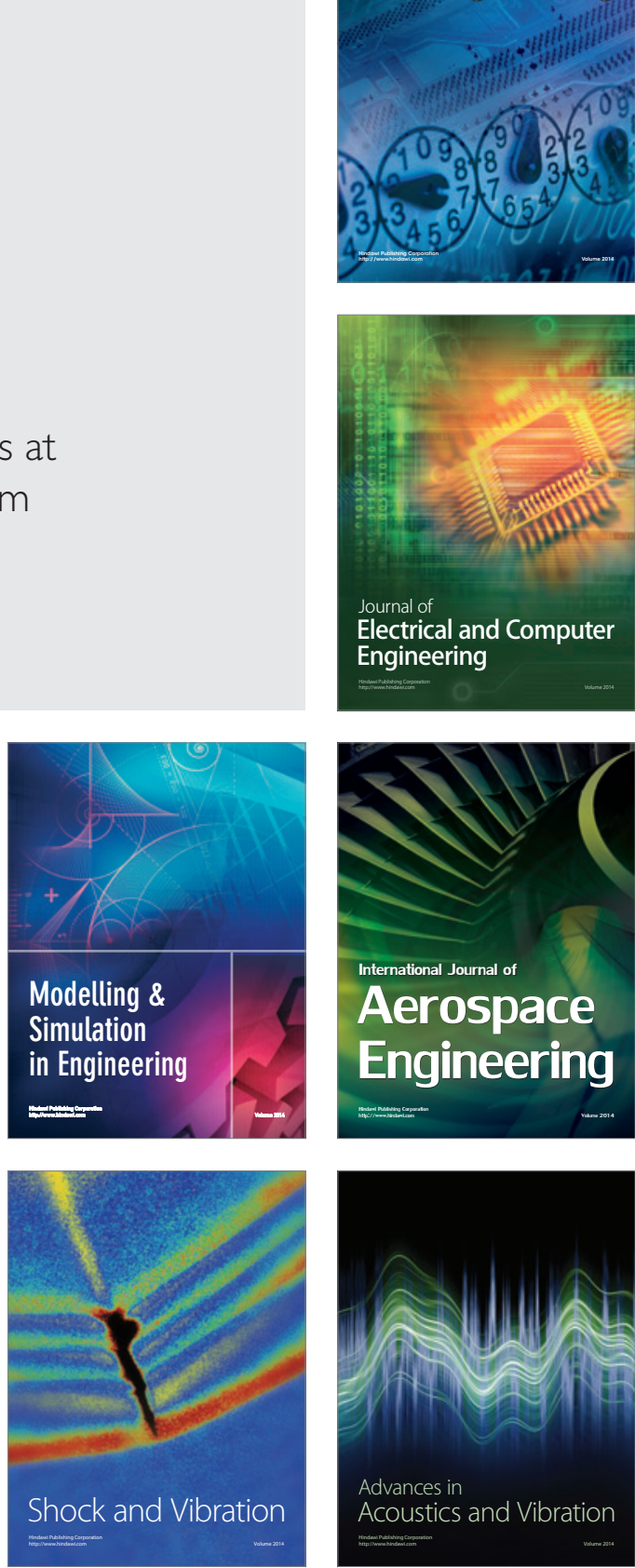